J. Korean Math. Soc. 51 (2014), No. 6, pp. 1105-1122

http://dx.doi.org/10.4134/JKMS.2014.51.6.1105

\title{
BIHARMONIC CURVES IN FINSLER SPACES
}

\author{
NiCOLETA Voicu
}

\begin{abstract}
Biharmonic curves are a generalization of geodesics, with applications in elasticity theory and computer science. The paper proposes a first study of biharmonic curves in spaces with Finslerian geometry, covering the following topics: a deduction of their equations, specific properties and existence of non-geodesic biharmonic curves for some classes of Finsler spaces. Integration of the biharmonic equation is presented for two concrete Finsler metrics.
\end{abstract}

\section{Introduction}

In elasticity theory, as well as in other domains, such as computer graphics and image processing, a classical problem is that of finding the extremal curves of the elastic energy (or bending energy) functional, given by the integral of the squared curvature $k^{2}$ of a curve with respect to its arc length $s$. Its extremal curves (called least-energy or elastic curves) are interpreted as the "smoothest" curves joining some given points or with given boundary conditions (see [9], [11]).

The Riemannian generalization of the elastic energy, called the bienergy, is defined as:

$$
E_{2}(c)=\frac{1}{2} \int_{c} \kappa^{2} d s,
$$

where $\kappa$ is the geodesic curvature of the curve $c$. Critical points of $E_{2}$, called biharmonic curves, are described by the equation:

$$
\nabla_{\dot{c}}^{3} \dot{c}=R\left(\dot{c}, \nabla_{\dot{c}} \dot{c}\right) \dot{c} .
$$

In Riemannian spaces, biharmonic curves and, more generally, biharmonic maps, were examined quite in detail (see [6], [8], [12], [13], [14], [17], [18], [19]). As a first remark, geodesics of $\nabla$ are always biharmonic (actually, they are minimum points for the bienergy), but the converse is generally not true.

\footnotetext{
Received July 23, 2013; Revised February 23, 2014.

2010 Mathematics Subject Classification. 53B40, 53C60, 31B30, 58E10.

Key words and phrases. Finsler space, bienergy, biharmonic curve.
} 
A non-harmonic biharmonic curve is called proper biharmonic. One of the most discussed related problems is the existence of proper biharmonic curves. We only mention here several results (see [13], [17], [18]):

- In the Euclidean space $\mathbb{R}^{n}$, every biharmonic curve is a segment of a straight line.

- On surfaces with nonpositive Gauss curvature, any biharmonic curve is a geodesic.

- In Riemannian spaces with nonpositive sectional curvature, every closed biharmonic curve is a geodesic.

Also, along any (unit speed) biharmonic curve, the geodesic curvature $\kappa$ is a constant.

Passing to Finsler spaces, the interest in biharmonicity is motivated, beyond purely mathematical reasons, by the potential of Finsler geometry for models in elasticity theory (see [5], [20]). In the present paper, we find the equations of biharmonic curves in Finsler spaces and study the Finslerian counterparts of the above mentioned results.

The paper is organized as follows. Section 2 is an introductory one, devoted to the necessary notions and results in Finsler geometry. In Section 3, we define the bienergy of a Finslerian curve and determine the equations of biharmonic curves. In Section 4, we show that Finslerian biharmonic curves have constant geodesic curvature. The next section investigates the problem of the existence of proper biharmonic curves in several special cases. For instance, we show that, to a difference from the Riemannian case, in dimension greater or equal to three, there exist flat (locally Minkowskian) Finsler spaces which admit proper biharmonic curves; also, we determine proper biharmonic curves for two concrete examples of Finsler metrics. In Section 6, we present a brief discussion of the obtained results.

\section{Finsler structures}

In this section, we present known notions and results in Finsler geometry to be used in the following. Consider a $\mathcal{C}^{\infty}$-smooth, $n$-dimensional differentiable manifold $M$ and denote by $(x, y):=\left(x^{i}, y^{i}\right)_{i=\overline{1, n}}$, the local coordinates on its tangent bundle $(T M, \pi, M)$. By ${ }_{, i}$ and ${ }_{i} i$ we will mean partial differentiation with respect $x^{i}$ and $y^{i}$, respectively; by $\Gamma(E)$ we will mean the module of the sections of some bundle $E$ over $M$.

A Finsler structure $([3],[5])$ on the manifold $M$ is a function $F: T M \rightarrow \mathbb{R}$ with the properties:

1) $F(x, y)$ is $\mathcal{C}^{\infty}$-smooth for $y \neq 0$ and continuous at $y=0$.

2) $F(x, \lambda y)=\lambda F(x, y), \forall \lambda>0$.

3) The Finslerian metric tensor:

$$
g_{i j}(x, y):=\frac{1}{2}\left(F^{2}(x, y)\right)_{\cdot i j}
$$

is positive definite for $y \neq 0$. 
By its dependence on the fiber coordinate $y$, the Finslerian metric tensor $g_{i j}$ "lives" on the tangent bundle $T M$, hence it is natural to perform the study on this bundle bee $^{1}$ (5], [15]).

The arc length of a curve $c$ on the Finsler space $(M, g)$ is given ([3]) by:

$$
l(c)=\int_{c} F(x, d x)
$$

A key ingredient in Finsler geometry, which considerably simplifies calculations, are adapted frames given by Ehresmann (nonlinear, [3], [5], [15]) connections on $T M$. The typical choice is the Cartan nonlinear connection $T T M=H T M \oplus V T M$, having the property that complete lifts to $T M$ of tangent vectors of unit speed geodesics of $M$ are always horizontal. The Cartan nonlinear connection is locally defined by the coefficients

$$
G^{i}{ }_{j}:=G^{i}{ }{ }_{j},
$$

where $G^{i}=G^{i}(x, y)$ are obtained from the equations of unit speed geodesics of $(M, F)$ :

$$
\frac{d y^{i}}{d s}+2 G^{i}(x, y)=0, \quad y=\dot{x}
$$

The (generally, nonholonomic) adapted basis on $\Gamma(T T M)$ is then given by:

$$
\left(\delta_{i}=\frac{\partial}{\partial x^{i}}-G_{i}^{j}(x, y) \frac{\partial}{\partial y^{j}}, \quad \dot{\partial}_{i}=\frac{\partial}{\partial y^{i}}\right)
$$

and its dual is: $\left(d x^{i}, \delta y^{i}=d y^{i}+G^{i}{ }_{j} d x^{j}\right)$. Any vector field $X$ on $T M$ can be decomposed as: $X=h X+v X, h X=X^{i} \delta_{i}, v X=\hat{X}^{i} \dot{\partial}_{i}$; its horizontal component $h X$ and its vertical component $v X$ are vector fields on $T M$.

In the presence of a nonlinear connection, vector fields on $M$ can be identified with horizontal vector fields on $T M$ via the horizontal lift

$$
l^{h}: \Gamma(T M) \rightarrow \Gamma(H T M), \quad \mathbf{X}=\mathbf{X}^{i} \partial_{i} \mapsto l^{h}(\mathbf{X})=\left(\mathbf{X}^{i} \circ \pi\right) \delta_{i} .
$$

The Finslerian metric tensor $g$ gives rise to a scalar product

$$
\langle X, Y\rangle=g_{i j} X^{i} Y^{j}
$$

of horizontal vector fields $X=X^{i} \delta_{i}, Y=Y^{j} \delta_{j} \in \Gamma(H T M)$.

The following (1,1)-type tensor fields on $T M$ :

$$
J=\dot{\partial}_{i} \otimes d x^{i}, \theta=\delta_{i} \otimes \delta y^{i},
$$

have the role of mapping horizontal vector fields into vertical ones and viceversa $\left(J\left(\delta_{i}\right)=\dot{\partial}_{i}, J\left(\dot{\partial}_{i}\right)=0 ; \theta\left(\dot{\partial}_{i}\right)=\delta_{i}, \theta\left(\delta_{i}\right)=0\right)$.

As covariant differentiation rule for tensors on $T M$, we will use the one given by the Cartan affine connection $D$ on $T M([5],[15])$ locally described by the coefficients:

(9) $D_{\delta_{k}} \delta_{j}=\Gamma_{j k}^{i} \delta_{i}, \quad D_{\delta_{k}} \dot{\partial}_{j}=\Gamma_{j k}^{i} \dot{\partial}_{i}, \quad D_{\dot{\partial}_{k}} \delta_{j}=C^{i}{ }_{j k} \delta_{i} D_{\dot{\partial}_{k}} \dot{\partial}_{j}=C^{i}{ }_{j k} \dot{\partial}_{i}$,

\footnotetext{
${ }^{1}$ Another natural variant is the pullback bundle $\pi^{*} T M$, used in [3], [16].
} 
where $\Gamma_{j k}^{i}=\Gamma_{j k}^{i}(x, y), C^{i}{ }_{j k}=C^{i}{ }_{j k}(x, y)$ are as follows:

$$
\Gamma_{j k}^{i}=\frac{1}{2} g^{i h}\left(\delta_{k} g_{h j}+\delta_{j} g_{h k}-\delta_{h} g_{j k}\right) ; \quad C_{j k}^{i}=\frac{1}{2} g^{i h} g_{h j \cdot k} .
$$

The Cartan connection is metrical and preserves by parallelism the horizontal and vertical distributions on $T T M$, i.e.,

$$
D_{X}(h Y)=h D_{X} Y, D_{X}(v Y)=v D_{X} Y, \quad \forall X, Y \in \Gamma(T T M) .
$$

Its torsion $\mathcal{T}$ has, in the adapted frame, the following nonzero local components:

(12) $\mathcal{T}(X, Y)=R^{i}{ }_{j k} \dot{\partial}_{i} \otimes d x^{k} \otimes d x^{j}+C^{i}{ }_{j k} \delta_{i} \otimes \delta y^{k} \otimes d x^{j}+P^{i}{ }_{j k} \dot{\partial}_{i} \otimes \delta y^{k} \otimes d x^{j}$, with $C^{i}{ }_{j k}$ as in (10) and:

$$
R^{i}{ }_{j k}=\delta_{k} G^{i}{ }_{j}-\delta_{j} G_{k}^{i}, \quad P^{i}{ }_{j k}=y^{l} C^{i}{ }_{j k \mid l},
$$

where $\left.\right|_{l}:=D_{\delta_{l}}$. The first of the three terms in (12) is the curvature of the nonlinear connection $N$ and the other two define two specific geometric objects to Finsler geometry:

2) the Cartan tensor ${ }^{2}: C(X, Y):=h \mathcal{T}(J X, Y), \forall X, Y \in \Gamma(H T M)$, in local writing: $C=C^{i}{ }_{j k} \delta_{i} \otimes d x^{k} \otimes d x^{j}$;

$3)$ the Landsberg tensor $P(X, Y):=\theta \mathcal{T}(J X, Y), \forall X, Y \in \Gamma(H T M)$, in local coordinates: $P:=P^{i}{ }_{j k} \delta_{i} \otimes d x^{k} \otimes d x^{j}$.

The Cartan and the Landsberg tensors obey similar properties:

i) the scalar products $\langle C(X, Y), W\rangle,\langle P(X, Y), W\rangle$ are totally symmetric in $X, Y, W \in \Gamma(H T M)$;

ii) transvection of $C$ and $P$ by $y$ provides zero, i.e.,

$$
C\left(l^{h}(y), \cdot\right)=0, P\left(l^{h}(y), \cdot\right)=0 .
$$

The curvature $\mathcal{R}$ of the Cartan connection $D$ also splits in the adapted basis into horizontal and vertical components. The local expressions of these components can be found, for instance, in [5], [15]; here, we will only mention some of their properties:

a) $\forall U, V \in \Gamma(T M): \mathcal{R}(v U, h V)\left(l^{h} y\right)=P(\theta U, h V) ; \mathcal{R}(v U, v V)\left(l^{h} y\right)=0$;

b) the $h(h h)$ component of $\mathcal{R}$ gives rise to the Jacobi endomorphism of $(M, F)([5])$ understood here as a $(1,1)$-type horizontal tensor:

$$
R(X)=\mathcal{R}\left(X, l^{h} y\right) l^{h} y, \quad \forall X \in \Gamma(H T M) ;
$$

in local writing, $R=R_{j}^{i} \delta_{i} \otimes d x^{j}, R_{j}^{i}=R_{k j}^{i} y^{k}$. The components $R_{i k}$ are symmetric, therefore,

$$
\langle R(X), Y\rangle=\langle R(Y), X\rangle, \quad \forall X, Y \in \Gamma(H T M) .
$$

\footnotetext{
${ }^{2}$ The Cartan tensor is a measure of the non-Riemannian character of the Finsler metric; the equality $C=0$ is equivalent to the fact that the metric is Riemannian.
} 
If the system $\left\{l^{h}(y), X\right\}$ is orthonormal, then $K(X):=\frac{1}{F^{2}}\langle R(X), X\rangle$ gives the flag curvature of $(M, F)([3])$, which represents an analogue of sectional curvature from Riemannian spaces.

\section{Particular cases:}

1) In Riemannian spaces, we have $g_{i j}=g_{i j}(x), C=0, P=0$, while the $h(h h)$ component $\mathcal{R}(h U, h V) h W(U, V, W \in \Gamma(T M))$ is, up to a horizontal lift, the Riemannian curvature of $(M, g)$.

2) Locally Minkowski spaces ("flat" Finsler spaces) are spaces in which there exists a local chart $\left(U, x^{i}\right)$ around any point $x \in M$ such that, in the induced coordinates on $T M, g$ does not depend on $x$, i.e., $g_{i j}=g_{i j}(y)$. In this case, $\Gamma_{j k}^{i}=0, R=0, P=0$, but, generally, $C \neq 0$. Geodesics of a locally Minkowski space are locally (in the above mentioned coordinate system) given by:

$$
x^{i}(t)=x_{0}^{i}+t a^{i}, \quad x_{0}^{i}, a^{i}: \text { const.; }
$$

such geodesics are called rectilinear ([1], p. 116).

3) Landsberg spaces are defined by the condition $P=0$.

\section{Bienergy of a curve and its first variation}

Consider a curve $c: I \rightarrow M, s \mapsto\left(x^{i}(s)\right.$ ) (where $I \subset \mathbb{R}$ is an interval), parametrized by its arc length and its lift $c^{\prime}: I \rightarrow T M, s \mapsto\left(x^{i}(s), \dot{x}^{i}(s)\right)$ to $T M$; that is, $c$ has unit Finslerian speed:

$$
F(x, \dot{x})=1 .
$$

Tangent vectors of $c^{\prime}$ are expressed in the adapted basis as:

$$
T^{\prime}:=c_{*}^{\prime}\left(\partial_{s}\right)=y^{i} \delta_{i}+\frac{\delta y^{i}}{d s} \dot{\partial}_{i}, \quad y^{i}=\dot{x}^{i}
$$

We will denote simply by $D:=D^{c^{\prime}}$ the pullback of the Cartan affine connection $D$ by $c^{\prime}$. Taking into account that $\Gamma^{i}{ }_{j k} y^{k}=G^{i}{ }_{j}([5],[15])$, the components of $T^{\prime}$ in the adapted basis can be re-expressed ${ }^{3}$ as:

$$
T:=h T^{\prime}=l^{h}(y), \quad v T^{\prime}=J\left(D_{\partial_{s}} T\right) .
$$

The energy of the curve $c$ is given $([16])$ by

$$
E_{1}(c)=\frac{1}{2} \int_{c}\langle T, T\rangle d s .
$$

Critical points of the energy, i.e., harmonic curves ([16]) are constant speed geodesics of $(M, F)$, described by:

$$
D_{\partial_{s}} T=0
$$

The horizontal vector field

$$
\tau(c):=D_{\partial_{s}} T
$$

\footnotetext{
${ }^{3}$ Here and in the following, covariant differentiation is made, [3], with "reference vector" $T$, i.e., in the expressions of the connection coefficients $\Gamma=\Gamma(x, y)$ and $C=C(x, y)$, we set $\left.y^{i}=\dot{x}^{i}\right)$.
} 
defined along the curve $c$, is called (by analogy with the Riemannian case) the tension of $c([16])$. The norm of the tension field, i.e., $\kappa=\sqrt{\left\langle D_{\partial_{s}} T, D_{\partial_{s}} T\right\rangle}$, gives the geodesic curvature of the curve $c$.

It appears as natural to define the bienergy as follows:

Definition 1. 1) The bienergy of a curve $c$ on a Finsler space $(M, F)$ is:

$$
E_{2}(c)=\frac{1}{2} \int_{c}\left\langle D_{\partial_{s}} T, D_{\partial_{s}} T\right\rangle d s .
$$

2) Critical points of the bienergy are called biharmonic curves of $(M, F)$.

There holds the following result:

Theorem 2. A curve $c$ on the Finsler space $(M, F)$ is biharmonic if and only if

(24) $\quad \tau_{2}(c):=D_{\partial_{s}}^{3} T+R\left(D_{\partial_{s}} T\right)-P\left(D_{\partial_{s}} T, D_{\partial_{s}} T\right)+C\left(D_{\partial_{s}} T, D_{\partial_{s}}^{2} T\right)=0$.

Proof. Let us consider smooth variations $c_{\varepsilon}(s)=\tilde{c}(\varepsilon, s), c_{0}(s)=c(s)$ with fixed endpoints of $c$ and their lifts $c_{\varepsilon}^{\prime}=\left(c_{\varepsilon}, \frac{d c_{\varepsilon}}{d s}\right)$ to $T M$. With the notations:

$$
\mathbf{T}^{\prime}:=\tilde{c}_{*}^{\prime}\left(\partial_{s}\right), \mathbf{V}^{\prime}:=\tilde{c}_{*}^{\prime}\left(\partial_{\varepsilon}\right) ; \quad \mathbf{T}:=h \mathbf{T}^{\prime}, \mathbf{V}:=h \mathbf{V}^{\prime},
$$

we have $v \mathbf{T}^{\prime}=J\left(D_{\partial_{s}} \mathbf{T}\right)$ and, at $\varepsilon=0$ :

$$
\left.T=\mathbf{T}\rfloor_{\varepsilon=0}, \quad V:=\mathbf{V}\right\rfloor_{\varepsilon=0} .
$$

Taking into account that the Cartan connection $D$ is metrical, we can write:

$$
\frac{d E_{2}}{d \varepsilon}\left(c_{\varepsilon}\right)=\frac{1}{2} \int_{c} \frac{d}{d \varepsilon}\left\langle D_{\partial_{s}} \mathbf{T}, D_{\partial_{s}} \mathbf{T}\right\rangle d s=\int_{c}\left\langle D_{\partial_{\varepsilon}} D_{\partial_{s}} \mathbf{T}, D_{\partial_{s}} \mathbf{T}\right\rangle d s .
$$

Further, since $D$ preserves the distributions generated by $N$ and (25),

$$
\begin{aligned}
D_{\partial_{\varepsilon}} D_{\partial_{s}} \mathbf{T} & =\mathcal{R}\left(\mathbf{V}^{\prime}, \mathbf{T}^{\prime}\right) \mathbf{T}+D_{\partial_{s}} D_{\partial_{\varepsilon}}\left(h \mathbf{T}^{\prime}\right) \\
& =\mathcal{R}\left(\mathbf{V}^{\prime}, \mathbf{T}^{\prime}\right) \mathbf{T}+D_{\partial_{s}}\left(h D_{\partial_{\varepsilon}} \mathbf{T}^{\prime}\right) \\
& =\mathcal{R}\left(\mathbf{V}^{\prime}, \mathbf{T}^{\prime}\right) \mathbf{T}+D_{\partial_{s}}\left(h \mathcal{T}\left(\mathbf{V}^{\prime}, \mathbf{T}^{\prime}\right)\right)+D_{\partial_{s}}^{2}\left(h \mathbf{V}^{\prime}\right) ;
\end{aligned}
$$

with $\mathbf{V}=h \mathbf{V}^{\prime}$ and substituting into (26), we get:

$$
\frac{d E_{2}}{d \varepsilon}=\int_{c}\left\langle\mathcal{R}\left(\mathbf{V}^{\prime}, \mathbf{T}^{\prime}\right) \mathbf{T}+D_{\partial_{s}}\left(h \mathcal{T}\left(\mathbf{V}^{\prime}, \mathbf{T}^{\prime}\right)\right)+D_{\partial_{s}}^{2} \mathbf{V}, D_{\partial_{s}} \mathbf{T}\right\rangle d s .
$$

Let us evaluate each term in the above integral. First of all, the curvature term is:

$$
\begin{aligned}
I_{1} & :=\int_{c}\left\langle\mathcal{R}\left(\mathbf{V}^{\prime}, \mathbf{T}^{\prime}\right) \mathbf{T}, D_{\partial_{s}} \mathbf{T}\right\rangle d s \\
& =\int_{c}\left\langle\mathcal{R}(\mathbf{V}, \mathbf{T}) \mathbf{T}+\mathcal{R}\left(v \mathbf{V}^{\prime}, \mathbf{T}\right) \mathbf{T}+\mathcal{R}\left(\mathbf{V}, v \mathbf{T}^{\prime}\right) \mathbf{T}+\mathcal{R}\left(v \mathbf{V}^{\prime}, v \mathbf{T}^{\prime}\right) \mathbf{T}, D_{\partial_{s}} \mathbf{T}\right\rangle d s .
\end{aligned}
$$

Along each of the curves $c_{\varepsilon}$, we have $\mathbf{T}=l^{h}(y)$; taking into account the properties of the curvature components, the terms $\left\langle\mathcal{R}\left(v \mathbf{V}^{\prime}, \mathbf{T}\right) \mathbf{T}, D_{\partial_{s}} \mathbf{T}\right\rangle$ and 
$\left\langle\mathcal{R}\left(v \mathbf{V}^{\prime}, v \mathbf{T}^{\prime}\right) \mathbf{T}, D_{\partial_{s}} \mathbf{T}\right\rangle$ vanish, while the two remaining ones can be written in a simpler form:

$$
\begin{aligned}
\left\langle\mathcal{R}(\mathbf{V}, \mathbf{T}) \mathbf{T}, D_{\partial_{s}} \mathbf{T}\right\rangle & =\left\langle R(\mathbf{V}), D_{\partial_{s}} \mathbf{T}\right\rangle \stackrel{(16)}{=}\left\langle R\left(D_{\partial_{s}} \mathbf{T}\right), \mathbf{V}\right\rangle \\
\left\langle\mathcal{R}\left(h \mathbf{V}^{\prime}, v \mathbf{T}^{\prime}\right) \mathbf{T}, D_{\partial_{s}} \mathbf{T}\right\rangle & \left.\left.=-\left\langle P\left(D_{\partial_{s}} \mathbf{T}, \mathbf{V}\right), D_{\partial_{s}} \mathbf{T}\right)\right\rangle=-\left\langle P\left(D_{\partial_{s}} \mathbf{T}, D_{\partial_{s}} \mathbf{T}\right), \mathbf{V}\right)\right\rangle
\end{aligned}
$$

we can therefore write:

$$
I_{1}=\int_{c}\left\langle R\left(D_{\partial_{s}} \mathbf{T}\right)-P\left(D_{\partial_{s}} \mathbf{T}, D_{\partial_{s}} \mathbf{T}\right), \mathbf{V}\right\rangle d s
$$

The second (torsion) term in (27), i.e.,

$$
I_{2}:=\int_{c}\left\langle D_{\partial_{s}}\left(h \mathcal{T}\left(\mathbf{V}^{\prime}, \mathbf{T}^{\prime}\right)\right), D_{\partial_{s}} \mathbf{T}\right\rangle d s
$$

will be integrated by parts:

$$
I_{2}=\int_{c}\left\{\partial_{s}\left\langle h \mathcal{T}\left(\mathbf{V}^{\prime}, \mathbf{T}^{\prime}\right), D_{\partial_{s}} \mathbf{T}\right\rangle-\left\langle\left(h \mathcal{T}\left(\mathbf{V}^{\prime}, \mathbf{T}^{\prime}\right)\right), D_{\partial_{s}}^{2} \mathbf{T}\right\rangle\right\} d s
$$

Since the variation has fixed endpoints, we have: $\int_{c} \partial_{s}\left\langle h \mathcal{T}\left(\mathbf{V}^{\prime}, \mathbf{T}^{\prime}\right), D_{\partial_{s}} \mathbf{T}\right\rangle d s=$ 0 ; using (12) and (14) in the evaluation of the remaining term, we are led to:

$$
I_{2}=\int_{c}\left\langle C\left(D_{\partial_{s}} \mathbf{T}, \mathbf{V}\right), D_{\partial_{s}}^{2} \mathbf{T}\right\rangle d s=\int_{c}\left\langle C\left(D_{\partial_{s}} \mathbf{T}, D_{\partial_{s}}^{2} \mathbf{T}\right), \mathbf{V}\right\rangle d s
$$

Finally, integrating twice by parts the third term in (27), i.e.,

$$
I_{3}:=\int_{c}\left\langle D_{\partial_{s}}^{2} \mathbf{V}, D_{\partial_{s}} \mathbf{T}\right\rangle d s
$$

we are led to:

$$
I_{3}:=\int_{c}\left\langle D_{\partial_{s}}^{3} \mathbf{T}, \mathbf{V}\right\rangle d s .
$$

Summing up and evaluating at $\varepsilon=0$, the first variation of the bienergy is:

$$
\begin{aligned}
\frac{d E_{2}}{d \varepsilon} & =I_{1}+I_{2}+I_{3} \\
& =\int_{c}\left\langle D_{\partial_{s}}^{3} T+R\left(D_{\partial_{s}} T\right)-P\left(D_{\partial_{s}} T, D_{\partial_{s}} T\right)+C\left(D_{\partial_{s}} T, D_{\partial_{s}}^{2} T\right), V\right\rangle d s,
\end{aligned}
$$

which proves the statement.

The vector field $\tau_{2}(c)$ in $(24)$ is called the bitension of $c$. In the following, we will also write the biharmonic equation (24) as:

$$
D_{\partial_{s}}^{3} T=\mathcal{A}
$$

where

$$
\mathcal{A}:=-R\left(D_{\partial_{s}} T\right)+P\left(D_{\partial_{s}} T, D_{\partial_{s}} T\right)-C\left(D_{\partial_{s}} T, D_{\partial_{s}}^{2} T\right) .
$$

Remark. Any unit speed geodesic on $M$ is biharmonic; a non-geodesic biharmonic curve will be called proper biharmonic. 


\section{Particular cases:}

1) If $M$ is a Riemannian space, then the equation of biharmonic curves reduces to:

$$
D_{\partial_{s}}^{3} T+R\left(D_{\partial_{s}} T\right)=0 \Leftrightarrow D_{\partial_{s}}^{3} T-\mathcal{R}\left(T, D_{\partial_{s}} T\right) T=0 .
$$

Up to a projection onto the base manifold, this is the known equation (2).

2) In locally Minkowski spaces, equation (24) becomes:

$$
D_{\partial_{s}}^{3} T+C\left(D_{\partial_{s}} T, D_{\partial_{s}}^{2} T\right)=0 .
$$

3) In Landsberg spaces, $\tau_{2}(c)=D_{\partial_{s}}^{3} T+R\left(D_{\partial_{s}} T\right)+C\left(D_{\partial_{s}} T, D_{\partial_{s}}^{2} T\right)$.

\section{Frenet frame and geodesic curvature}

We will suppose that, along $c$, the vectors $\left\{T, D_{\partial_{s}} T, D_{\partial_{s}}^{2} T, \ldots, D_{\partial_{s}}^{n-1} T\right\}$ are linearly independent; in particular, this implies that $D_{\partial_{s}} T \neq 0$, i.e., $c$ is nongeodesic. Similarly to Riemannian geometry ([4], [7]) we get the Frenet frame along $c$ as the orthonormalization of these vectors, obeying the relations:

$$
\left\{\begin{array}{l}
e_{1}=T=l^{h}(y) \\
D_{\partial_{s}} e_{1}=\kappa_{1} e_{2} \\
D_{\partial_{s}} e_{2}=-\kappa_{1} e_{1}+\kappa_{2} e_{3} \\
D_{\partial_{s}} e_{i}=-\kappa_{i-1} e_{i-1}+\kappa_{i} e_{i+1}, \quad i=2, \ldots, n-1 \\
\cdots \\
D_{\partial_{s}} e_{n}=-\kappa_{n-1} e_{n-1}
\end{array}\right.
$$

the only difference in the Finslerian case is that here, $e_{1}, \ldots, e_{n}$ are regarded as sections of the bundle $\left(c^{\prime}\right)^{-1}(H T M)$. The generalized curvatures $\kappa_{1}, \ldots, \kappa_{n-1}$ are positive numbers ${ }^{4}$.

Remarks. 1) The first curvature $\kappa_{1}$, i.e.,

$$
\kappa_{1}:=\sqrt{\left\langle D_{\partial_{s}} T, D_{\partial_{s}} T\right\rangle}=\kappa
$$

is the geodesic curvature of $c$.

2 ) In the case when for some $s \in I$, the vector $T(s)$ and its covariant derivatives are linearly independent just up to some order $r, 1 \leq r<n-1$, only the vectors $e_{1}, \ldots, e_{r+1}$ of the Frenet frame can be constructed.

Using (31), the expressions of the covariant derivatives $D_{\partial_{s}}^{i} T, i=\overline{1,3}$ in the Frenet frame are:

$$
\left\{\begin{array}{l}
D_{\partial_{s}} T=\kappa_{1} e_{2} \\
D_{\partial_{s}}^{2} T=-\kappa_{1}^{2} e_{1}+\kappa_{1}^{\prime} e_{2}+\kappa_{1} \kappa_{2} e_{3} \\
D_{\partial_{s}}^{3} T=-3 \kappa_{1} \kappa_{1}^{\prime} e_{1}+\left(\kappa_{1}^{\prime \prime}-\kappa_{1}^{3}-\kappa_{1} \kappa_{2}^{2}\right) e_{2}+\left(2 \kappa_{1}^{\prime} \kappa_{2}+\kappa_{1} \kappa_{2}^{\prime}\right) e_{3}+\kappa_{1} \kappa_{2} \kappa_{3} e_{4} .
\end{array}\right.
$$

\footnotetext{
${ }^{4}$ While all constructions of the Frenet frame in Riemannian geometry seem to agree upon the sign of $\kappa_{1}, \ldots, \kappa_{n-2}$, the sign of the top curvature $\kappa_{n-1}$ appears to be a matter of convention. There exist approaches such as the one we chose to generalize, in [4], [7], in which $\kappa_{n-1}$ is positive (which also works on non-orientable manifolds), as well as constructions in which the sign of $\kappa_{n-1}$ is variable, e.g., [10].
} 
Substituting (33) into the biharmonic equation (29) and using the fact that the Cartan tensor $C$ vanishes on $e_{1}$, we have, on one hand:

$$
\mathcal{A}=-\kappa_{1} R\left(e_{2}\right)+\kappa_{1}^{2} P\left(e_{2}, e_{2}\right)-\kappa_{1} \kappa_{1}^{\prime} C\left(e_{2}, e_{2}\right)-\kappa_{1}^{2} \kappa_{2} C\left(e_{2}, e_{3}\right) .
$$

On the other hand, taking the scalar product of the third equality (33) with $e_{i}, i=\overline{1, n}$ and noticing that $\left\langle\mathcal{A}, e_{1}\right\rangle=0$, we find:

Proposition 3. A unit speed curve $c: I \rightarrow M$ is biharmonic if and only if

$$
\left\{\begin{array}{l}
\kappa_{1} \kappa_{1}^{\prime}=0 \\
\kappa_{1}^{\prime \prime}-\kappa_{1}^{3}-\kappa_{1} \kappa_{2}^{2}=\left\langle\mathcal{A}, e_{2}\right\rangle \\
2 \kappa_{1}^{\prime} \kappa_{2}+\kappa_{1} \kappa_{2}^{\prime}=\left\langle\mathcal{A}, e_{3}\right\rangle \\
\kappa_{1} \kappa_{2} \kappa_{3}=\left\langle\mathcal{A}, e_{4}\right\rangle \\
0=\left\langle\mathcal{A}, e_{i}\right\rangle, \quad i \geq 5
\end{array}\right.
$$

The assumption that $c$ is non-geodesic means that $\kappa_{1} \neq 0$, which, by (35), gives us $\kappa_{1}^{\prime}=0$. That is, if $c$ is a proper biharmonic curve parametrized by arc length, then:

$$
\kappa_{1}=\text { const. } \neq 0 \text {. }
$$

Since in the case when $\kappa_{1}=0$ (i.e., $c$ is a geodesic), the curve is trivially biharmonic, we immediately get:

Theorem 4. Along any biharmonic curve of a Finsler space, the geodesic curvature $\kappa_{1}=\left\|D_{\partial_{s}} T\right\|$ is constant.

A similar result is known to hold in Riemannian geometry (see [2], [6], [14], $[18])$.

\section{Existence of proper biharmonic curves}

\subsection{Closed biharmonic curves}

We will assume in the following that $c$ is a smooth closed biharmonic curve. Since in this case, $c$ and its derivatives coincide at its endpoints, we can write:

$$
0=\int_{c} \frac{1}{2} \frac{d^{2}}{d s^{2}}\left\langle D_{\partial_{s}} T, D_{\partial_{s}} T\right\rangle d s=\int_{c}\left\{\left\langle D_{\partial_{s}}^{3} T, D_{\partial_{s}} T\right\rangle+\left\langle D_{\partial_{s}}^{2} T, D_{\partial_{s}}^{2} T\right\rangle\right\} d s .
$$

Using the biharmonic equation, this is:

$$
0=\int_{c}\left\{\left\langle\mathcal{A}, D_{\partial_{s}} T\right\rangle+\left\langle D_{\partial_{s}}^{2} T, D_{\partial_{s}}^{2} T\right\rangle\right\} d s
$$

Let us evaluate $\int_{c}\left\langle\mathcal{A}, D_{\partial_{s}} T\right\rangle d s$. By virtue of the total symmetry of $C$, the term involving it in the mentioned integral is:

$$
\int_{c}\left\langle-C\left(D_{\partial_{s}} T, D_{\partial_{s}}^{2} T\right), D_{\partial_{s}} T\right\rangle d s=-\int_{c}\left\langle C\left(D_{\partial_{s}} T, D_{\partial_{s}} T\right), D_{\partial_{s}}^{2} T\right\rangle d s .
$$


Since the curve is closed, we have $\int_{c} \partial_{s}\left\langle\left(C\left(D_{\partial_{s}} T, D_{\partial_{s}} T\right)\right), D_{\partial_{s}} T\right\rangle=0$, that is, integration by parts in (37) leads to:

$$
\begin{aligned}
& \int_{c}\left\langle-C\left(D_{\partial_{s}} T, D_{\partial_{s}} T\right), D_{\partial_{s}}^{2} T\right\rangle d s \\
= & \int_{c}\left\langle D_{\partial_{s}}\left(C\left(D_{\partial_{s}} T, D_{\partial_{s}} T\right)\right), D_{\partial_{s}} T\right\rangle d s \\
= & \left.\int_{c}\left\langle\left(D_{\partial_{s}} C\right)\left(D_{\partial_{s}} T, D_{\partial_{s}} T\right), D_{\partial_{s}} T\right\rangle+2\left\langle C\left(D_{\partial_{s}} T, D_{\partial_{s}}^{2} T\right), D_{\partial_{s}} T\right\rangle\right\} d s .
\end{aligned}
$$

Consider the following tensor acting on horizontal vector fields:

$$
\tilde{C}(X, Y, Z):=\left(D_{J X} C\right)(Y, Z), \quad \forall X, Y, Z \in \Gamma(H T M) ;
$$

then, using the total symmetry of $C$ and (13), we can write:

$$
\begin{aligned}
& \int_{c}\left\langle-C\left(D_{\partial_{s}} T, D_{\partial_{s}}^{2} T\right), D_{\partial_{s}} T\right\rangle d s \\
= & \frac{1}{3} \int_{c}\left\langle\left(D_{\partial_{s}} C\right)\left(D_{\partial_{s}} T, D_{\partial_{s}} T\right), D_{\partial_{s}} T\right\rangle d s \\
= & \frac{1}{3} \int_{c}\left\{\left\langle P\left(D_{\partial_{s}} T, D_{\partial_{s}} T\right)+\tilde{C}\left(D_{\partial_{s}} T, D_{\partial_{s}} T, D_{\partial_{s}} T\right), D_{\partial_{s}} T\right\rangle\right\} d s .
\end{aligned}
$$

Substituting into (36), we finally have:

$$
0=\int_{c}\left\{\mathcal{F}\left(D_{\partial_{s}} T\right)+\left\langle D_{\partial_{s}}^{2} T, D_{\partial_{s}}^{2} T\right\rangle\right\} d s
$$

where

(40) $\mathcal{F}(X):=\left\langle-R(X)+\frac{4}{3} P(X, X)+\frac{1}{3} \tilde{C}(X, X, X), X\right\rangle, \quad X \in \Gamma(H T M)$.

Assume that $\mathcal{F}(X)$ is nonnegative for any $X$. Then from (39), it follows that $D_{\partial_{s}}^{2} T=0$. We will show that the latter equality implies that $c$ is harmonic. Indeed, taking the integral:

$$
0=\int_{c} \frac{d}{d s}\left\langle T, D_{\partial_{s}} T\right\rangle d s=\int_{c}\left\{\left\langle D_{\partial_{s}} T, D_{\partial_{s}} T\right\rangle+\left\langle T, D_{\partial_{s}}^{2} T\right\rangle\right\} d s,
$$

the last term in the right hand side is zero, hence $\left\langle D_{\partial_{s}} T, D_{\partial_{s}} T\right\rangle=0$. By virtue of the positive definiteness of $g$, this leads to:

$$
D_{\partial_{s}} T=0 \text {, }
$$

i.e., $c$ is harmonic. We have thus proved:

Proposition 5. If, in the Finsler space $(M, F)$, the operator

$$
\mathcal{F}: \Gamma(H T M) \rightarrow \mathbb{R}, \quad \mathcal{F}(X)=\left\langle-R(X)+\frac{4}{3} P(X, X)+\frac{1}{3} \tilde{C}(X, X, X), X\right\rangle
$$

takes only nonnegative values, then any closed biharmonic curve on $M$ is a geodesic. 
Particular case: If $g$ is Riemannian, then

$$
\mathcal{F}(X)=\langle-R(X), X\rangle=\left\langle-\mathcal{R}\left(X, l^{h} y\right) l^{h} y, X\right\rangle=-\operatorname{Riem}_{g}\left(y, \pi_{*} X\right) \cdot \sigma,
$$

where Riem $_{g}$ is the sectional curvature of $(M, g)$ and $\sigma:=g(y, y) g\left(\pi_{*} X, \pi_{*} X\right)-$ $\left(g\left(y, \pi_{*} X\right)\right)^{2} \geq 0$. Thus, we obtain in this case a known result $([13])$ : any closed biharmonic curve on a Riemannian manifold with nonpositive sectional curvature, is harmonic.

\subsection{2-dimensional Finsler spaces}

If $(M, F)$ is an oriented Finsler surface, the Frenet equations (31) reduce to:

$$
e_{1}=T, \quad D_{\partial_{s}} e_{1}=\kappa_{1} e_{2}, \quad D_{\partial_{s}} e_{2}=-\kappa_{1} e_{1}
$$

and the equations of proper biharmonic curves become:

$$
\left\{\begin{array}{l}
\kappa_{1} \kappa_{1}^{\prime}=0 ; \\
\kappa_{1}^{\prime \prime}-\kappa_{1}^{3}=\left\langle-\kappa_{1} R\left(e_{2}\right)+\kappa_{1}^{2} P\left(e_{2}, e_{2}\right)-\kappa_{1} \kappa_{1}^{\prime} C\left(e_{2}, e_{2}\right), e_{2}\right\rangle,
\end{array}\right.
$$

that is, $\kappa_{1} \neq 0$ is a constant and:

$$
\kappa_{1}^{2}=\left\langle R\left(e_{2}\right)-\kappa_{1} P\left(e_{2}, e_{2}\right), e_{2}\right\rangle .
$$

Since the left hand side of the above equality is always positive, we have a series of immediate consequences.

1) In Landsberg spaces, (41) becomes similar to the corresponding relation in Riemannian spaces ([17]):

$$
\kappa_{1}^{2}=\left\langle R\left(e_{2}\right), e_{2}\right\rangle=K\left(e_{2}\right) .
$$

It follows:

Proposition 6. In 2-dimensional Landsberg spaces with nonpositive (not necessarily constant) flag curvature, any biharmonic curve is a geodesic.

2) In locally Minkowski spaces, where $R=0, P=0$, (41) becomes: $\kappa_{1}=0$, i.e., any biharmonic curve is, again, a geodesic. Taking into account (17), we obtain:

Proposition 7. In 2-dimensional locally Minkowski spaces, any biharmonic curve is a rectilinear geodesic.

\subsection{Locally Minkowski spaces}

Assume now that $(M, F)$ is a locally Minkowski space with $\operatorname{dim} M \geq 3$. Then, the biharmonic equation (24) reduces to:

$$
D_{\partial_{s}}^{3} T+C\left(D_{\partial_{s}} T, D_{\partial_{s}}^{2} T\right)=0 .
$$

Let us choose a local chart in which $g_{i j}=g_{i j}(y)$ and denote

$$
D_{\partial_{s}} T=: \tau^{i} \delta_{i}
$$


the components of the tension of $c$ in the adapted basis; with this notation, (42) is read as:

$$
\frac{d}{d s} \frac{D \tau^{i}}{d s}+2 C_{h k}^{i} \tau^{h} \frac{D \tau^{k}}{d s}=0
$$

Lowering indices by $g_{i j}$ and taking into account that $g_{i j \cdot k}=2 C_{i j k}, \frac{d g_{i j}}{d s}=$ $2 C_{i j k} \tau^{k}$, this is:

We get the first integrals:

$$
\frac{d}{d s}\left(g_{i h} \frac{D \tau^{i}}{d s}\right)=\frac{D \tau_{h}}{d s}=0 .
$$

$$
\frac{D \tau_{h}}{d s}=\lambda_{h}, \quad \lambda_{h}=\text { const. }
$$

So far, we have seen that two classes of locally Minkowski spaces, namely:

- Euclidean spaces $([17])$;

- 2-dimensional spaces, do not to admit proper biharmonic curves. Using (44), we will prove that such a result cannot be extended to arbitrary locally Minkowskian Finsler spaces.

Proposition 8. There exist locally Minkowski spaces of dimension at least three, which admit proper biharmonic curves.

Proof. The space $\mathbb{R}^{3}$ equipped with the Randers-type Minkowski norm ([3]):

$$
F(y):=\sqrt{\left(y^{1}\right)^{2}+\left(y^{2}\right)^{2}+\left(y^{3}\right)^{2}}+b y^{3}
$$

(with $b \in(0,1))$ admits proper biharmonic curves. The detailed calculation is presented below, in Section 5.4.2.

\subsection{Examples}

5.4.1. A 2-dimensional Randers-Numata metric. Let us consider the unit disk $M=\left\{x \in \mathbb{R}^{2} \mid \delta_{i j} x^{i} x^{j}<1\right\}$, equipped with the Randers-type metric:

$$
F(x, y)=\sqrt{\delta_{i j} y^{i} y^{j}}+\delta_{i j} x^{i} y^{j}=: \alpha+\delta_{i j} x^{i} y^{j}
$$

and look for its proper biharmonic curves. For this metric, we know ([3, p. 70]) that:

$$
G^{i}=\frac{\alpha^{2}}{2 F} y^{i}
$$

(note: our $G^{i}$ is as in [1], [5], [15], i.e., half of the one in [3]) and its flag curvature $K:=K(X)$ does not depend on the vector field $X \perp l^{h}(y)$; it is given by:

$$
K=\frac{3}{4 F^{4}}\left(\delta_{i j} y^{i} y^{j}\right)^{2}=\frac{3}{4 F^{4}} \alpha^{4} .
$$

Moreover, the space is projectively flat, i.e., it has rectilinear geodesics.

The following equalities on $(M, F)$ can be checked by direct computation:

$$
g_{i j}=F F_{\cdot i j}+F_{\cdot i} F_{\cdot j}=F \alpha_{\cdot i j}+F_{\cdot i} F_{\cdot j}
$$




$$
\begin{aligned}
& F_{, i}=\alpha \alpha_{\cdot i}, \quad F_{\cdot i j}=\alpha_{\cdot i j}, F_{\cdot i j k}=\alpha_{\cdot i j k} ; \\
& C_{i j k}=\frac{1}{4} F_{\cdot i j k}^{2}=\frac{1}{2}\left(F_{\cdot i} F_{\cdot j k}+F_{\cdot j} F_{\cdot i k}+F_{\cdot k} F_{\cdot i j}+F F_{\cdot i j k}\right) ; \\
& \left(\alpha^{2}\right)_{\cdot i j k}=0 \Rightarrow \alpha \alpha_{\cdot i j k}=-\left(\alpha_{\cdot i} \alpha_{\cdot j k}+\alpha_{\cdot j} \alpha_{\cdot i k}+\alpha_{\cdot k} \alpha_{\cdot i j}\right) .
\end{aligned}
$$

Along a unit speed curve $c: I \rightarrow M, s \mapsto\left(x^{i}(s)\right)$ (more precisely, along its lift $s \mapsto c^{\prime}(s):=\left(x^{i}(s), y^{i}(s):=\dot{x}^{i}(s)\right)$ to $\left.T M\right)$ there hold the relations:

(53) $\quad F(x, y)=\|T\|=1, \quad F_{\cdot i}=\frac{g_{i j} y^{j}}{F} ; \quad F_{\cdot i} \tau^{i}=\frac{1}{F}\left\langle T, D_{\partial_{s}} T\right\rangle=0 ;$

$$
\alpha_{\cdot i} y^{i}=\alpha, \quad \alpha_{\cdot i j} y^{j}=0 \quad \alpha_{\cdot i} \tau^{i}=\alpha^{\prime}+\frac{\alpha^{3}}{F},
$$

where $\alpha^{\prime}:=d \alpha / d s=y^{i} \delta_{i} \alpha+\tau^{i} \alpha_{. i}$ and $\tau^{i}$ are as in (43). The tension can be expressed as:

$$
\tau^{i}=\frac{d y^{i}}{d s}+\Gamma_{j k}^{i} y^{j} y^{k}=\frac{d y^{i}}{d s}+G^{i}{ }_{j} y^{j}=\frac{d y^{i}}{d s}+2 G^{i}=\frac{d y^{i}}{d s}+\frac{\alpha^{2}}{F} y^{i} .
$$

In the following, we will write the equation (41) of proper biharmonic curves as:

$$
\left\{\begin{array}{l}
g_{i j} \tau^{i} \tau^{j}=\kappa_{1}^{2}(=\text { const. } \neq 0) \\
-\kappa_{1}^{4}=\left\langle-R\left(D_{\partial_{s}} T\right), D_{\partial_{s}} T\right\rangle+\left\langle P\left(D_{\partial_{s}} T, D_{\partial_{s}} T\right), D_{\partial_{s}} T\right\rangle
\end{array}\right.
$$

Let us examine the first equation (56). Using (49), (53), (55) and (54), this condition becomes:

$$
\kappa_{1}^{2}=F \alpha_{\cdot i j} \tau^{i} \tau^{j}=F \alpha_{\cdot i j} \frac{d y^{i}}{d s} \frac{d y^{j}}{d s} .
$$

We have: $\alpha \cdot 11=\frac{\left(y^{2}\right)^{2}}{\alpha^{3}}, \alpha \cdot 12=\frac{-y^{1} y^{2}}{\alpha^{3}}, \alpha \cdot 22=\frac{\left(y^{1}\right)^{2}}{\alpha^{3}}$ and thus,

$$
\kappa_{1}^{2}=\frac{F\left(\dot{y}^{2} y^{1}-\dot{y}^{1} y^{2}\right)^{2}}{\alpha^{3}}=F \alpha\left(\frac{d}{d s}\left(\arctan \frac{y^{2}}{y^{1}}\right)\right)^{2} .
$$

Passing to (Euclidean) polar coordinates $y^{1}=\alpha \cos \theta, y^{2}=\alpha \sin \theta$ in the plane $\left(y^{1}, y^{2}\right)$, we have $\theta=\arctan \frac{y^{2}}{y^{1}}+$ const. and the first equation (56) can be finally written as:

$$
\theta=\kappa_{1} \int(F \alpha)^{-1 / 2} d s
$$

Consider now the second equation (56); the first term in its right hand side is:

$$
\left\langle-R\left(D_{\partial_{s}} T\right), D_{\partial_{s}} T\right\rangle=-\kappa_{1}^{2}\left\langle R\left(e_{2}\right), e_{2}\right\rangle=-\kappa_{1}^{2} F^{2} K=\frac{-3 \kappa_{1}^{2}}{4 F^{2}} \alpha^{4} .
$$

In order to calculate the second term $\left\langle P\left(D_{\partial_{s}} T, D_{\partial_{s}} T\right), D_{\partial_{s}} T\right\rangle=P_{i j k} \tau^{i} \tau^{j} \tau^{k}$, we make use of (13):

$$
P_{i j k}=y^{l} C_{i j k \mid l}=y^{l}\left(C_{i j k, l}-G_{l}^{h} C_{i j k \cdot h}-\Gamma_{i l}^{h} C_{h j k}-\Gamma_{j l}^{h} C_{i h k}-\Gamma_{k l}^{h} C_{i j h}\right) .
$$


First of all, from: $C_{i j k, l}=\frac{1}{4}\left(F_{, l}^{2}\right)_{\cdot i j k}=\frac{1}{2}\left(F F_{, l}\right)_{\cdot i j k}=\frac{1}{4}\left(F \alpha_{\cdot l}^{2}\right)_{\cdot i j k}$, it follows, with the help of (50) and (52), that $y^{l} C_{i j k, l}=0$.

Then, taking into account that $y^{l} \Gamma^{h}{ }_{i l}=G^{h}{ }_{i}, y^{l} G^{h}{ }_{l}=2 G^{h}$, the expression (47) of $G^{i}$ and of the fact that $C_{i j k}$ is homogeneous of degree -1 in $y$, we obtain:

$$
P_{i j k}=-\frac{\alpha^{2}}{2 F} C_{i j k}
$$

Substituting (51) in (60), contracting with $\tau^{i} \tau^{j} \tau^{k}$ and then using (53), we find: $P_{i j k} \tau^{i} \tau^{j} \tau^{k}=-\frac{\alpha^{2}}{4} F_{\cdot i j k} \tau^{i} \tau^{j} \tau^{k}=-\frac{\alpha^{2}}{4} \alpha_{\cdot i j k} \tau^{i} \tau^{j} \tau^{k}$. Finally, by (52), (54) and (57), we find:

$$
\left\langle P\left(D_{\partial_{s}} T, D_{\partial_{s}} T\right), D_{\partial_{s}} T\right\rangle=\frac{3 \kappa_{1}^{2}}{4}\left(\frac{\alpha \alpha^{\prime}}{F}+\frac{\alpha^{4}}{F^{2}}\right) .
$$

Using the latter and (59), together with $F=1$ in the second equation (56), this becomes:

$$
\frac{3 \alpha \alpha^{\prime}}{4}=-\kappa_{1}^{2}
$$

with the solution:

$$
\alpha=(\mu s+\nu)^{1 / 2},
$$

where $\mu:=-\frac{8 \kappa_{1}^{2}}{3}$ and $\nu \in \mathbb{R}$ are constants. Once we have calculated $\alpha$, we are able to calculate $\theta$ (from (58)), as:

$$
\theta=\kappa_{1} \int \alpha^{-1 / 2} d s=\frac{4 \kappa_{1}}{3 \mu}(\mu s+\nu)^{3 / 4}+\gamma,
$$

where $\gamma \in \mathbb{R}$. Substituting into $y^{1}=\alpha \cos \theta, y^{2}=\alpha \sin \theta$ and integrating with respect to $s$, we can finally state:

Proposition 9. Proper biharmonic curves of $(M, F)$ are given, in the Finslerian natural parametrization, by:

$$
\left\{\begin{array}{l}
x^{1}=\int \alpha \cos \theta d s=\int(\mu s+\nu)^{1 / 2} \cos \left(\frac{4 \kappa_{1}}{3 \mu}(\mu s+\nu)^{3 / 4}+\gamma\right) d s \\
x^{2}=\int \alpha \sin \theta d s=\int(\mu s+\nu)^{1 / 2} \sin \left(\frac{4 \kappa_{1}}{3 \mu}(\mu s+\nu)^{3 / 4}+\gamma\right) d s
\end{array}\right.
$$

5.4.2. A Randers-Minkowski norm on $\mathbb{R}^{3}$. Let us find proper biharmonic curves of the Minkowski norm:

$$
F(y)=\sqrt{\left(y^{1}\right)^{2}+\left(y^{2}\right)^{2}+\left(y^{3}\right)^{2}}+b y^{3}=: \alpha+\beta
$$

on $M=\mathbb{R}^{3}$. That is, we will look for non-constant $y=\dot{x}$ obeying the biharmonic equation and $F(y)=1$.

Let us denote: $\beta=b_{i} y^{i}, b_{i}:=b \delta_{3 i}$. The metric tensor $g_{i j}$ is given ([3]) by:

$$
g_{i j}=\frac{F}{\alpha}\left(\delta_{i j}-\alpha_{\cdot i} \alpha_{\cdot j}\right)+F_{\cdot i} F_{\cdot j} .
$$


Also, there hold relations (49)-(52) and, along any unit speed curve $c$ on $(M, F)$, we have:

$$
\begin{gathered}
\tau^{i}=\frac{d y^{i}}{d s}, \quad F_{\cdot i} \tau^{i}=F^{\prime}=0, \alpha_{\cdot i} \tau^{i}=\alpha^{\prime} ; \\
\alpha_{\cdot i j} \tau^{i} \tau^{j}=F_{\cdot i j} \tau^{i} \tau^{j}=\frac{\kappa_{1}^{2}}{F}, \quad F_{\cdot j} \frac{d \tau^{j}}{d s}=-\frac{\kappa_{1}^{2}}{F}, \\
\alpha_{\cdot i j} \tau^{j}=\frac{1}{\alpha}\left(\delta_{i j} \tau^{j}-\alpha_{i} \alpha^{\prime}\right), \quad \alpha_{\cdot j} \frac{d \tau^{j}}{d s}=\alpha^{\prime \prime}-\frac{\kappa_{1}^{2}}{F} ;
\end{gathered}
$$

Note that along arbitrary unit speed curves, $\kappa_{1}^{2}:=g_{i j} \tau^{i} \tau^{j}$ is not necessarily constant.

Biharmonic curves are given by (44), i.e.,

$$
g_{i j} \frac{D \tau^{j}}{d s}=\lambda_{i}, \quad \lambda_{i}=\text { const. }
$$

In the following, we will express these equations in terms of the Euclidean metric $\delta_{i j}$ and of the derivatives of $\alpha$.

By (62), (64), (65), we have:

$$
g_{i j} \frac{d \tau^{j}}{d s}=\frac{F}{\alpha} \delta_{i j} \frac{d \tau^{j}}{d s}+\left(\frac{\kappa_{1}^{2}}{\alpha}-\frac{F \alpha^{\prime \prime}}{\alpha}\right) \alpha_{\cdot i}-\frac{\kappa_{1}^{2}}{F} F_{\cdot i} .
$$

For the second term in (66), we get, with the help of (50), (51), (63), (64):

$$
C_{i j k} \tau^{j} \tau^{k}=\frac{1}{2}\left(\frac{\kappa_{1}^{2}}{F} F_{\cdot i}+F \alpha_{\cdot i j k} \tau^{j} \tau^{k}\right)
$$

and further, using (52), (65):

$$
C_{i j k} \tau^{j} \tau^{k}=\frac{\kappa_{1}^{2}}{2 F} F_{\cdot i}+\left(\frac{F\left(\alpha^{\prime}\right)^{2}}{\alpha^{2}}-\frac{\kappa_{1}^{2}}{2 \alpha}\right) \alpha_{\cdot i}-\frac{F \alpha^{\prime}}{\alpha^{2}} \delta_{i j} \tau^{j}
$$

Adding (67) and (68), equations (44) become:

(69) $\left.\frac{F}{\alpha} \delta_{i j} \frac{d \tau^{j}}{d s}-\frac{F \alpha^{\prime}}{\alpha^{2}} \delta_{i j} \tau^{j}+\left(\frac{F\left(\alpha^{\prime}\right)^{2}}{\alpha^{2}}-\frac{F \alpha^{\prime \prime}}{\alpha}+\frac{\kappa_{1}^{2}}{2 \alpha}-\frac{\kappa_{1}^{2}}{2 F}\right)\right) \alpha_{\cdot i}=\lambda_{i}+\frac{\kappa_{1}^{2}}{2 F} b_{i}$.

Let us notice some more equalities, valid along any unit speed curve:

$$
\begin{gathered}
\alpha \cdot i y^{i}=\alpha, \quad \delta_{i j} y^{i} \tau^{j}=\frac{1}{2} \frac{d \alpha^{2}}{d s}=\alpha \alpha^{\prime}, \quad \delta_{i j} \tau^{i} \tau^{j}=\frac{\alpha \kappa_{1}^{2}}{F}+\left(\alpha^{\prime}\right)^{2}, \\
\delta_{i j} y^{i} \frac{d \tau^{j}}{d s}=\alpha \alpha^{\prime \prime}-\frac{\kappa_{1}^{2} \alpha}{F}, \quad \delta_{i j} \tau^{i} \frac{d \tau^{j}}{d s}=\frac{\alpha^{\prime}}{2 F} \kappa_{1}^{2}+\frac{\alpha \kappa_{1} \kappa_{1}^{\prime}}{F}+\alpha^{\prime} \alpha^{\prime \prime},
\end{gathered}
$$

where, in the third relation (70), we have used (62). Transvecting now the three equations (69) in turn by $y^{i}, \tau^{i}$ and $\delta^{i k} \lambda_{k}$ which can be proven with not too much difficulty to represent, along any proper biharmonic curve, the components of three linearly independent vectors and using (70), (71) together 
with $\alpha_{\cdot i} \tau^{i}=\alpha^{\prime}$, they take the form:

(72)

$$
\left\{\begin{array}{l}
\lambda_{i} y^{i}=-\kappa_{1}^{2} \\
\lambda_{i} \tau^{i}=\kappa_{1} \kappa_{1}^{\prime} \\
\left.\frac{F}{\alpha} \lambda_{j} \frac{d \tau^{j}}{d s}-\frac{F \alpha^{\prime}}{\alpha^{2}} \lambda_{j} \tau^{j}+\left(\frac{F\left(\alpha^{\prime}\right)^{2}}{\alpha^{2}}-\frac{F \alpha^{\prime \prime}}{\alpha}+\frac{\kappa_{1}^{2}}{2 \alpha}-\frac{\kappa_{1}^{2}}{2 F}\right)\right) \delta^{i j} \alpha_{\cdot i} \lambda_{j}=-\kappa_{1}^{2} \gamma,
\end{array}\right.
$$

where $\gamma:=-\kappa_{1}^{-2} \delta^{i j}\left(\lambda_{i}+\frac{\kappa_{1}^{2}}{2 F} b_{i}\right) \lambda_{j}$.

Differentiating the first equation (72) by $s$, we have: $\lambda_{i} \tau^{i}=-2 \kappa_{1} \kappa_{1}^{\prime}$, which, substituted into the second one yields:

$$
\kappa_{1}=\text { const. }
$$

(which was found in Section 5.1. to be true along any biharmonic curve). Thus, $\gamma$ is a constant and we have: $\lambda_{i} \tau^{i}=0 \Rightarrow \lambda_{i} \frac{d \tau^{i}}{d s}=0$. Further, noticing that $\delta^{i j} \alpha_{\cdot i}=\frac{y^{j}}{\alpha}$, the third equation (72) becomes:

$$
\frac{1}{\alpha}\left(\frac{F\left(\alpha^{\prime}\right)^{2}}{\alpha^{2}}-\frac{F \alpha^{\prime \prime}}{\alpha}+\frac{\kappa_{1}^{2}}{2 \alpha}-\frac{\kappa_{1}^{2}}{2 F}\right)=\gamma
$$

taking into account that, along $c, F \equiv 1, \alpha$ is given by:

$$
(\ln \alpha)^{\prime \prime}+\frac{\kappa_{1}^{2}}{2 \alpha}-\frac{\kappa_{1}^{2}}{2}-\gamma \alpha=0
$$

which obviously admits non-constant solutions.

Let $\alpha$ be a non-constant solution of (74). Then, substituting $\alpha=1-b y^{3}$, we get a non-constant value for $y^{3}=\dot{x}^{3}$. Further, $y^{1}=\dot{x}^{1}$ and $y^{2}=\dot{x}^{2}$ can be obtained from the relations $F(y)=1, \lambda_{i} y^{i}=-\kappa_{1}^{2}$.

\section{Conclusions}

In the present paper, we extend the notion of bienergy functional to curves on Finsler spaces and deduce the equations of biharmonic curves as the EulerLagrange equations attached to this functional; these equations turn out to involve, besides the flag curvature (which is the analogue of the curvature term in the Riemannian biharmonic equations), two specific, non-Riemannian quantities, namely, the Cartan tensor and the Landsberg tensor of the space. Still, just as in Riemannian spaces, all geodesics are biharmonic curves, but the converse is generally not true.

A brief analysis in the Frenet frame shows that, though the equation of biharmonic curves becomes more complicated compared to its Riemannian counterpart, a basic result in Riemannian biharmonicity theory remains true in our case, namely, any Finslerian biharmonic curve has constant geodesic curvature.

Further, we focus on the conditions under which the implication biharmonic $\rightarrow$ geodesic becomes true. The first case to be investigated is the one of smooth closed curves. In the particular case of Riemannian spaces, this turns out to be the known condition of nonpositive sectional curvature; still, in the general 
Finslerian case, this condition is more complicated, involving the Cartan and Landsberg tensors.

A special attention is paid to two-dimensional Finsler spaces (Section 5.2). We prove that, in dimension two, in: 1) Landsberg spaces with nonpositive flag curvature and 2) flat (i.e., locally Minkowski) Finsler spaces, any biharmonic curve is a geodesic.

Further, in Subsection 5.3., we investigate higher dimensional locally Minkowski spaces. Here, we find a first integral of the biharmonic equation and show that, unlike Euclidean spaces, flat Finsler spaces of dimension at least three can admit proper unit speed biharmonic curves.

Two examples: a 2-dimensional projectively flat space and a 3-dimensional locally Minkowski Finsler space, both admitting proper biharmonic curves, are given in Section 5.4 .

\section{References}

[1] P. L. Antonelli, R. S. Ingarden, and M. Matsumoto, The Theory of Sprays and Finsler Spaces with Applications in Physics and Biology, Kluwer Acad. Publ., 1993.

[2] A. Balmus, Biharmonic Maps and Submanifolds, Geom. Balkan Press, 2009.

[3] D. Bao, S. S. Chern, and Z. Shen, An Introduction to Riemann-Finsler Geometry, Graduate Texts in Mathematics, Vol 200, Springer, 2000.

[4] D. E. Blair, Riemannian Geometry of Contact and Symplectic Manifolds, 2nd edn., Progress in Mathematics, Vol. 203, Birkhauser, Boston, MA, 2010.

[5] I. Bucataru and R. Miron, Finsler-Lagrange Geometry - Applications to Dynamical Systems, Ed. Acad. Romane, Bucharest, 2007.

[6] R. Caddeo, S. Montaldo, C. Oniciuc, and P. Piu, The Euler-Lagrange method for biharmonic curves, Mediterr. J. Math. 3 (2006), no. 3-4, 449-465.

[7] C. Calin and M. Crasmareanu, Slant curves and particles in three-dimensional warped products and their Lancret invariants, Bull. Aust. Math. Soc. 88 (2013), no. 1, 128-142.

[8] D. Fetcu, Biharmonic Legendre curves in Sasakian space forms, J. Korean Math. Soc. 45 (2008), no. 2, 393-404.

[9] J. M. Glass, Smooth-curve interpolation: a generalized spline-fit procedure, BIT 6 (1966), $277-293$.

[10] E. Gutkin, Curvatures, volumes and norms of derivatives for curves in Riemannian manifolds, J. Geom. Phys. 61 (2011), no. 11, 2147-2161.

[11] B. K. P. Horn, The curve of least energy, ACM Trans. Math. Software 9 (1983), no. 4, 441-460.

[12] J. I. Inoguchi and J. E. Lee, Biminimal curves in 2-dimensional space forms, Commun. Korean Math. Soc. 27 (2012), no. 4, 771-780.

[13] G. Jiang, 2-harmonic maps and their first and second variational formulas, Note Mat. 28 (2009), 209-232.

[14] S. Maeta, $k$-harmonic maps into a Riemannian manifold with constant sectional curvature, Proc. Amer. Math. Soc. 140 (2012), no. 5, 1835-1847.

[15] R. Miron and M. Anastasiei, The Geometry of Lagrange Spaces: Theory and Applications, Kluwer Acad. Publ., 1994.

[16] X. Mo, An Introduction to Finsler Geometry, World Scientific, 2006.

[17] S. Montaldo and C. Oniciuc, A short survey on biharmonic maps between Riemannian manifolds, Rev. Un. Mat. Argentina 47 (2006), no. 2, 1-22.

[18] J. Monterde, Surfaces with a family of nongeodesic biharmonic curves, Rend. Mat. Appl. (7) 28(2) (2008), 123-131. 
[19] C. Oniciuc, Biharmonic submanifolds in space forms, Habil. Thesis, Univ. "Al. I. Cuza", Iasi, Romania, 2012.

[20] T. Yajima, K. Yamasaki, and H. Nagahama, Finsler metric and elastic constants for weak anisotropic media, Nonlinear Anal. Real World Appl. 12 (2011), no. 6, 3177-3184.

Faculty of Mathematics and Computer Science

Transilvania University

50, Iuliu Maniu Str., Brasov, Romania

E-mail address: nico.voicu@unitbv.ro 